\title{
Quantitative assessment of skin layers absorption and skin reflectance spectra simulation in the visible and near-infrared spectral regions
}

\author{
Igor V Meglinski ${ }^{1}$ and Stephen $\mathbf{J}$ Matcher $^{2}$ \\ ${ }^{1}$ School of Engineering, Cranfield University, Cranfield, MK43 0AL, UK \\ ${ }^{2}$ School of Physics, University of Exeter, Exeter, EX4 4QL, UK \\ E-mail: i.meglinski@cranfield.ac.uk and s.j.matcher@exeter.ac.uk
}

\begin{abstract}
We have simulated diffuse reflectance spectra of skin by assuming a wavelength-independent scattering coefficient for the different skin tissues and using the known wavelength dependence of the absorption coefficient of oxyand deoxyhaemoglobin and water. A stochastic Monte Carlo method is used to convert the wavelength-dependent absorption coefficient and wavelengthindependent scattering coefficient into reflected intensity. The absorption properties of skin tissues in the visible and near-infrared spectral regions are estimated by taking into account the spatial distribution of blood vessels, water and melanin content within distinct anatomical layers. The geometrical peculiarities of skin histological structure, degree of blood oxygenation and the haematocrit index are also taken into account. We demonstrate that when the model is supplied with reasonable physical and structural parameters of skin, the results of the simulation agree reasonably well with the results of in vivo measurements of skin spectra.
\end{abstract}

Keywords: Monte Carlo (MC) simulation, optical properties of skin, nearinfrared spectroscopy (NIR), skin blood content

\section{Introduction}

The quantified monitoring and localization of chromophores in biological tissues using optical/near-infrared (NIR) spectroscopy is a technologically challenging task. The ultimate aim of this technique is the production of tomographic maps of tissue haemoglobin concentration and saturation. Much theoretical and experimental progress has been made in this field over the last ten years, with iterative image reconstruction techniques utilizing 
time- or frequency-resolved measurements emerging as the most promising approach (Matcher 2002). Optical spectroscopy of the skin is a potentially useful tool to study disturbances in oxygenation in, e.g., venous ulceration.

The use of optical fibres allows the detection of diffusely reflected radiation within a given observation angle at a certain point on the medium surface. This permits control of the measurement volume, which is of particular importance for probing of biological tissues. We use a small source-detector optical fibre separation $(200-800 \mu \mathrm{m})$ in order to maximize the signal contribution of the capillary loops. These lie about $100 \mu \mathrm{m}$ under the surface and are of particular interest as they are the primary site of nutrient transport. Recently, we showed that for such a range of the fibre-optic probe spacing the main part of the detected signal becomes localized in the upper skin layers, which include the capillary loops (Meglinsky and Matcher 2001a, Meglinskii and Matcher 2001b). However, the extraction from the obtained attenuation spectrum of the absolute chromophore concentrations within the tissues is still complicated in this case due to multiple scattering within the tissues and reflections at tissue boundaries. Multi-linear regression provides a convenient tool for converting changes in light attenuation at several wavelengths into corresponding values of chromophores concentration (Matcher 2002). To validate this conversion approach we have generated simulated skin reflectance spectra. Validation of the algorithm can then be done by comparing the chromophore concentrations extracted from the simulated reflectance spectrum with the actual chromophore concentrations used to perform the spectrum simulation.

In this paper, we demonstrate that when our computational skin model is supplied with reasonable physical and structural parameters of internal tissues the results of the simulation agree reasonably well with the results of in vivo measurements of skin reflectance spectra.

\section{Methods}

\subsection{Monte Carlo simulation}

Theoretical modelling of light propagation in biological media is usually based on transport theory, or the diffusion approximation (Farrell et al 1992, Kienle and Patterson 1997), to the Boltzmann transport equation (Ishimaru 1978). Stochastic numerical Monte Carlo (MC) modelling has many advantages over analytical models (Cox and Durian 2001). Over the last decade a number of MC algorithms of optical radiation propagation in biological media have been developed for various applications (Wilson and Adam 1983, Bonner et al 1987, Keijzer et al 1989, Flock et al 1989, Prahl et al 1989, Yaroslavsky and Tuchin 1992, Wang et al 1995, Okada et al 1997, Meglinsky and Matcher 2001a). We employed an MC technique that combines the statistical weight scheme and real photon paths simulation (Meglinsky and Matcher 2001a, Meglinskii and Matcher 2001b). This approach avoids the energy conservation problem that occurs in the 'Russian roulette' approach (Keijzer et al 1989, Flock et al 1989, Prahl et al 1989, Yaroslavsky and Tuchin 1992, Wang et al 1995). Here, we briefly describe the MC model that we have developed to predict the spectra obtained using our fibre-optic reflectance probe (see figure 1).

The simulation is based on modelling a large number of possible trajectories of the photon packets from the site of photon injection in the medium (source $S$ ) to the site where the photons leave the medium (detector D) (figure 2). Modelling of the trajectory of an individual photon packet consists of the following sequence of steps: generation of photon path lengths, generation of scattering events and handling of Fresnel reflections/refractions on the medium surface and on internal layer boundaries. 


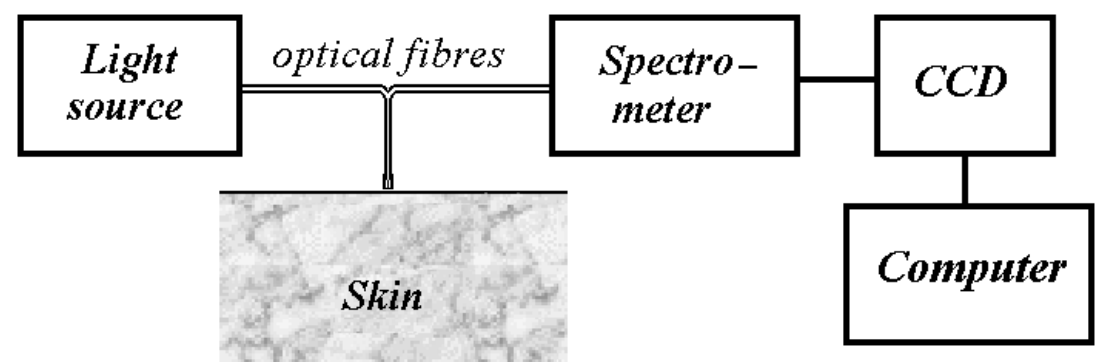

Figure 1. Schematic representation of the experimental set-up used for the non-invasive measurements of the diffuse reflectance spectra of skin. A $100 \mathrm{~W}$ quartz halogen lamp (L.O.T.ORIEL Ltd, Model 77501) is used as an optical/NIR source of light projected onto the skin surface by an optical fibre bundle. Another optical fibre separated from the source fibre by a short distance collects diffusely reflected light and delivers it to the detector. A SPEX 270M spectrograph/monochromator with a $1024 \times 256$ CCD camera (Wright Instruments Ltd) used for high speed spectral measurements, where one dimension is responsible for wavelength and the other for the intensity of detected scattered light. The detecting signal is processed by a personal computer (PC), where the spectral curve can be fitted with a multi-linear regression algorithm (Matcher 2002).

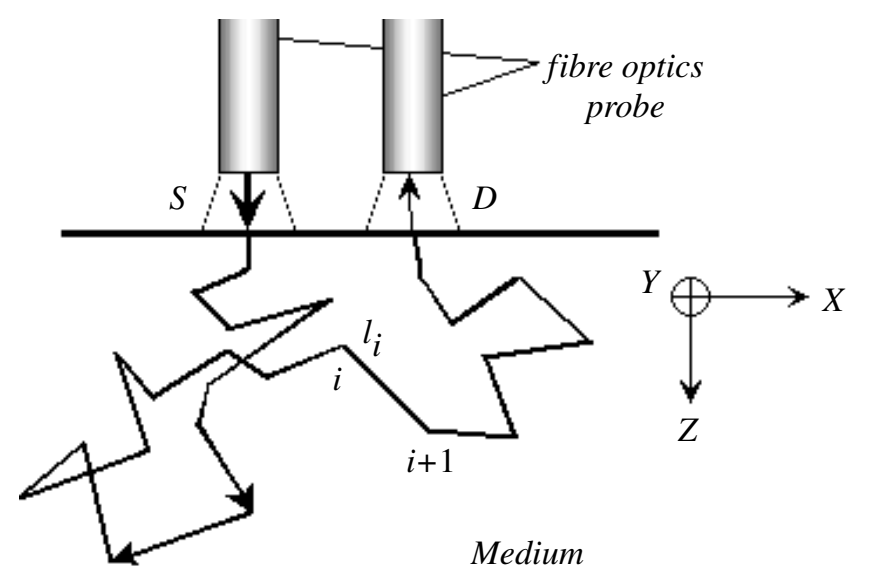

Figure 2. Photon random trajectory along which a photon travels from the source area $S$ to the detector $\mathrm{D}$ in a semi-infinite randomly inhomogeneous highly scattering medium. The Cartesian coordinate system $(X Y Z)$ is employed to specify the position of a photon in the modelling medium. Here, $l_{i}$ is the length of the $i$ th random step during which the photon moves from the $i$ th to $(i+1)$ th scattering event.

The photon path length $l$ can take on any positive values with the probability density function (Sobol' 1974):

$$
p(l)=\mu_{\mathrm{s}} \exp \left(-\mu_{\mathrm{s}} l\right) \exp \left(-\mu_{\mathrm{a}} l\right)+\mu_{\mathrm{a}} \exp \left(-\mu_{\mathrm{a}} l\right) \exp \left(-\mu_{\mathrm{s}} l\right)
$$

where $\mu_{\mathrm{s}}$ and $\mu_{\mathrm{a}}$ are the scattering and absorption coefficients, respectively. Here, the first term on the right-hand side describes the probability of photon scattering and its reduction due to medium absorption. The second term describes the probability of photon absorption in the medium reduced by scattering. In terms of optical diagnostics, the main interest lies in the detected photons rather than the photons lost and absorbed in the medium. Our simulation scheme involves first generating photon trajectories for the case of an absorptionfree medium; absorption is then handled by statistically re-weighting the detected photons. 
Hence we describe the photon path length probability density function (1) by the first term only. Thus, the path length that a photon packet moves at the $i$ th step (figure 2 ) is modelled as

$$
l_{i}=-\frac{\ln (\xi)}{\mu_{\mathrm{s}}}
$$

where $\xi$ is a uniformly distributed random number between 0 and 1 .

The scattering event is then simulated by generating two random propagation angles $\varphi$ and $\theta$, polar and azimuth, which describe the new direction in which the photon packet then travels. These angles are characterized by a Henyey-Greenstein scattering phase function (Henyey and Greenstein 1941):

$$
p(\theta)=\frac{1}{4 \pi} \frac{1-g^{2}}{\left(1+g^{2}-2 g \cos \theta\right)^{\frac{3}{2}}}
$$

where $g$ is the anisotropy factor equal to the average cosine of the scattering angle $\theta$.

The specular and internal reflection on the medium boundary is taken into account by splitting the photon packet into the reflected and the transmitted parts, the weights of which are attenuated as

$$
W=\left(1-R\left(\alpha_{i}\right)\right)\left(1-R_{\text {in }}\right) W_{0}\left[\prod_{p=1}^{M} R_{p}\left(\alpha_{i}\right)\right]
$$

where $W_{0}$ is the initial weight of the photon packet, $M$ is the number of photon packet reflections/refractions on the medium boundary, $R_{\text {in }}$ is the Fresnel coefficient relating to the initial reflection on the medium boundary surface, when the photon packet enters the medium, $R(\alpha)$ is the Fresnel reflection coefficient:

$$
R(\alpha)= \begin{cases}\frac{\left(n-n_{0}\right)^{2}}{\left(n+n_{0}\right)^{2}} & \text { if } \quad \alpha=0 \\ \frac{1}{2}\left[\frac{\sin ^{2}\left(\alpha-\alpha_{\mathrm{t}}\right)}{\sin ^{2}\left(\alpha+\alpha_{\mathrm{t}}\right)}+\frac{\tan ^{2}\left(\alpha-\alpha_{\mathrm{t}}\right)}{\tan ^{2}\left(\alpha+\alpha_{\mathrm{t}}\right)}\right] & \text { if } \quad 0<\alpha<\sin ^{-1}\left(\frac{n}{n_{0}}\right) \\ 1 & \text { if } \quad \sin ^{-1}\left(\frac{n}{n_{0}}\right)<\alpha<\frac{\pi}{2} .\end{cases}
$$

Here $\alpha$ and $\alpha_{\mathrm{t}}$ are the angles of incidence and transmittance of photon packets on the medium surface, respectively, and $n$ and $n_{0}$ are the refractive indices on either side of the interface.

To decide whether a photon is reflected or transmitted at internal refractive-indexmismatched boundaries we follow the procedure used in the 'Russian roulette' approach (Keijzer et al 1989, Prahl et al 1989, Yaroslavsky and Tuchin 1992, Wang et al 1995), i.e. compare a random number $\xi_{B}$ uniformly distributed between 0 and 1 to the value of $R(\alpha)$ calculated using (5) and allow either reflection or transmission of the photon if $\xi_{B}$ is smaller or bigger than $R(\alpha)$, respectively.

The process of tracing a photon packet is continued until it is either detected, leaves the medium or its path length exceeds a threshold value. The total number of detected photon packets is usually $10^{6}-10^{7}$. The individual trajectory of each photon packet that arrives at the detector through the non-absorbing medium $\left(\mu_{\mathrm{a}}=0\right)$ is stored in a data file. Then, we include the absorption of the medium by recalculating the statistical weight of each photon packet according to its stored trajectory as

$$
W_{\mathrm{D}}=W_{j} \exp \left(-\sum_{i=1}^{N_{j}} \mu_{\mathrm{a}} l_{i}\right)
$$


Here, $N_{j}$ is the number of scattering events undergone by the $j$ th photon packet during its random walk, $l_{i}$ is the photon packet path length at the $i$ th scattering event as calculated using (2) and $W_{j}$ is the statistical weight of the jth photon packet reaching the detector area in the absorption-free medium $\left(\mu_{\mathrm{a}}=0\right)$. Such an approach of separate simulation of scattering and absorption agrees with the microscopic Beer-Lambert law, and allows the rapid recalculation of the reflected intensity for many different values of $\mu_{\mathrm{a}}$ :

$$
I(\lambda)=\sum_{q=1}^{N_{\mathrm{ph}}}\left(\prod_{j=1}^{M_{q}} R_{j}(\alpha)\right) \exp \left(-\sum_{k=1}^{N_{q}} \mu_{\mathrm{a} k}(\lambda) l_{k}\right) .
$$

Here, $N_{\mathrm{ph}}$ is the number of total number of detected photon packets, $N_{q}$ and $M_{q}$ are respectively the number of scattering events and photon reflections/refractions on the medium boundary undergone by the $q$ th photon packet during its random walk from the source to detector area.

\subsection{Computational model of the skin}

2.2.1. 3D modelling of skin structure. As an object of investigation, skin represents a complex heterogeneous medium, where the spatial distribution of blood and chromophores are variable with depth. Nonetheless, it is possible to define anatomical regions in skin, where the skin cell structure, chromophore content and blood concentration are roughly constant. This allows us to approximate skin as a multi-layered medium. Following earlier work aimed at computational modelling of the skin (Keijzer et al 1989, Prahl et al 1989, Yaroslavsky and Tuchin 1992, Tuchin 1992, Wang et al 1995, Svaasand et al 1995, Verkruysse et al 1999, Meglinsky and Matcher 2001a, Meglinskii and Matcher 2001b) we have simulated skin as a three-dimensional half-infinite media divided into seven layers.

The first layer corresponds to the layer of desquamating flattened dead cells mainly containing keratin, which is $20 \mu \mathrm{m}$ thick, and known as the stratum corneum. The second layer, which we call living epidermis, is $80 \mu \mathrm{m}$ thick and is assumed to contain primarily living cells: a fraction of dehydrated cells, laden cells with keratohyalin granules, columnar cells, and also melanin dust, small melanin granules and melanosomes (Odland 1991). Significantly, this layer is not directly perfused with blood and is thus dependent on passive oxygen diffusion from the lower lying dermis for its vitality. Given the inhomogeneous distribution of blood vessels and capillaries within the skin (Renkin et al 1984, Ryan 1991) we subdivide the dermis into four different layers, with different blood volumes. These layers are papillary dermis (150 $\mu \mathrm{m}$ thick), upper blood net dermis (80 $\mu \mathrm{m}$ thick), reticular dermis (1500 $\mu \mathrm{m}$ thick) and deep blood net dermis (100 $\mu \mathrm{m}$ thick). The deepest layer in our model is subcutaneous fat (6000 $\mu \mathrm{m}$ thick). Of course, some variability in thickness is expected from region to region of the body and between individuals, and histological evidence suggests this can be of order $30-40 \%$. However, for our present study we have taken these values for layer thickness to be typical for adults. Moreover, taking into account these variations and the different cell structures of skin tissues we represent the interfaces between the skin layers as quasi-random periodic surfaces (Meglinsky and Matcher 2001a, Meglinskii and Matcher 2001b):

$$
\begin{gathered}
B_{k}(x, y)=Z_{0 k}\left(x_{0}, y_{0}\right)+\left(A_{k x} \sin \left(\omega_{k x} x+\phi_{k x}\right)+a_{k x} \sin \left(\omega_{k y}^{\prime} y+\phi_{k x}^{\prime}\right)\right) \\
\times\left(A_{k y} \sin \left(\omega_{k y} y+\phi_{k y}\right)+a_{k y} \sin \left(\omega_{k y}^{\prime} y+\phi_{k y}^{\prime}\right)\right)
\end{gathered}
$$

where $B_{k}(x, y)$ is the depth of the $k$ th layer at the point $(x, y), Z_{0 k}\left(x_{0}, y_{0}\right)$ is the mean depth of the boundary, $A_{k x}, A_{k y}, a_{k x}, a_{k y}$ are the amplitude coefficients, $\omega_{k x}, \omega_{k y}, \omega_{k x}^{\prime}, \omega_{k y}^{\prime}$ are the scale lengths of the roughness and $\phi_{k x}, \phi_{k y}, \phi_{k x}^{\prime}, \phi_{k y}^{\prime}$ are arbitrary phase offsets, respectively, in the $x$ and $y$ directions. The rough boundaries between the layers of different refractive 


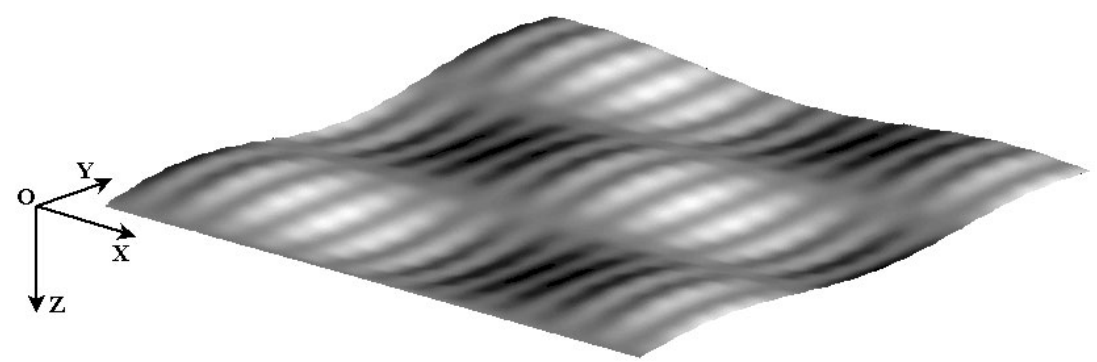

Figure 3. An example of a quasi-random periodic surface representing the interfaces between the dermal layers.

Table 1. The values of the parameters defined in equation (8) and used in our simulation.

\begin{tabular}{lllllr}
\hline$k$ & Boundary between layers & $A_{k x}, A_{k y}(\mu \mathrm{m})$ & $\left(\frac{\pi}{\omega}\right)_{k x}(\mu \mathrm{m})$ & $\left(\frac{\pi}{\omega}\right)_{k y}(\mu \mathrm{m})$ & $Z_{0 k}(\mu \mathrm{m})$ \\
\hline 0 & Air-stratum corneum (skin surface) & 2 & 100 & 150 & 0 \\
1 & Stratum corneum-living epidermis & 2.5 & 80 & 80 & 20 \\
2 & Living epidermis-papillary dermis & 20 & 50 & 45 & 100 \\
3 & Papillary dermis-upper blood net dermis & 2 & 20 & 40 & 250 \\
4 & Upper blood net dermis-dermis & 2 & 20 & 50 & 330 \\
5 & Dermis-deep blood net dermis & 2 & 20 & 50 & 1830 \\
6 & Deep blood net dermis-subcutaneous fat & 5 & 20 & 50 & 1910 \\
7 & Subcutaneous fat-other tissues & 5 & 25 & 30 & 8000 \\
\hline
\end{tabular}

indices can play a significant role in the distribution of photons within the medium (Lu et al 2000, Meglinsky and Matcher 2001a, Meglinskii and Matcher 2001b). The values of the parameters presented in table 1 produce boundaries (see figure 3) that are comparable to the observed structure of real skin (Stenn 1988, Odland 1991, Holbrook 1991, Corcuff et al 1993, Serup and Jemec 1995).

2.2.2. Modelling of the optical properties of skin layers. The blood content of tissues can vary both in amount and in distribution (Renkin et al 1984, Ryan 1991). The volume fraction of dermis occupied by blood vessels is usually in the range 1-20\%, and varies in apparent blood content in the range $2-12 \%$. On the other hand, the skin is quite fibrous and the apparent average blood content is quite low. If one assumes that the blood is uniformly distributed in skin, then the average blood content would be about $0.2 \%$. However, the reality is that the blood is concentrated in the venous plexus at a concentration probably in the $2-10 \%$ range within a layer $50-100 \mu \mathrm{m}$ in thickness. This is one reason why we subdivide the dermis into four different layers: papillary dermis, upper blood net dermis, reticular dermis and deep blood net dermis. These layers are approximate, but illustrate that the spatial distribution of blood in a heterogeneous tissue is an important factor influencing light transport in the tissue, as has been discussed widely (Jacques 1996, Verkruysse et al 1999, Tuchin 2000).

Thus we determine the spectrum of the absorption coefficient in the visible and nearinfrared spectral region for each layer by taking into account the spatial distribution of blood vessels, water and melanin within the skin tissues:

$$
\mu_{\mathrm{a}}(\lambda)=\sum_{i=1}^{m}\left(\mu_{\mathrm{a}}^{(i)}(\lambda) C_{i} \prod_{j=1}^{i-1}\left(1-C_{j}\right)\right)+\mu_{\mathrm{a}}^{(0)}(\lambda) \prod_{i=1}^{m}\left(1-C_{i}\right)
$$


Table 2. The parameters used in the calculation of the absorption coefficients of skin layers ${ }^{\mathrm{a}}$, and other optical properties of the layers used in the simulation.

\begin{tabular}{lllllll}
\hline$k$ & Name of layer & $C_{\text {blood }}$ & $C_{\mathrm{H}_{2} \mathrm{O}}$ & $\mu_{s}\left(\mathrm{~mm}^{-1}\right)$ & $g$ & $n$ \\
\hline 1 & Stratum corneum & 0 & 0.05 & 100 & 0.86 & 1.5 \\
2 & Living epidermis & 0 & 0.2 & 45 & 0.8 & 1.34 \\
3 & Papillary dermis & 0.04 & 0.5 & 30 & 0.9 & 1.4 \\
4 & Upper blood net dermis & 0.3 & 0.6 & 35 & 0.95 & 1.39 \\
5 & Reticular dermis & 0.04 & 0.7 & 25 & 0.8 & 1.4 \\
6 & Deep blood net dermis & 0.1 & 0.7 & 30 & 0.95 & 1.38 \\
7 & Subcutaneous fat & 0.05 & 0.7 & 5 & 0.75 & 1.44 \\
\hline
\end{tabular}

${ }^{\mathrm{a}} C_{\text {blood }}$ estimated from a number of literature reports (Chapman 1980, Stenn 1988, Odland 1991, Holbrook 1991, Renkin et al 1984, Ryan 1991, Jacques 1996), $C_{\mathrm{H}_{2} \mathrm{O}}$ evaluated from (Chapman 1980, Potts 1985).

where $C_{i}$ is the volume fraction of the $i$ th absorber (i.e. blood, water or melanin) in the given skin layer, $m$ is the total number of absorbers contained in the layer; $\mu_{\mathrm{a}}^{(i)}(\lambda)$ is the absorption coefficient of the $i$ th absorber; $\mu_{\mathrm{a}}^{(0)}(\lambda)$ is the absorption coefficient caused by the intrinsic absorption of the medium free of any absorbers, defined as (Saidi 1992, Jacques 1996)

$$
\mu_{\mathrm{a}}^{(0)}(\lambda)=7.84 \times 10^{7} \times \lambda^{-3.255} .
$$

Including in (9) skin blood oxygen saturation $S$, and total volume fraction of haemoglobin in blood $\gamma$, the absorption coefficients of a dermal layer $\mu_{\mathrm{a}}^{\text {layer }}$ can be represented as

$$
\begin{gathered}
\mu_{\mathrm{a}}^{\text {layer }}(\lambda)=(1-S) \gamma C_{\text {blood }} \mu_{\mathrm{a}}^{\mathrm{Hb}}(\lambda)+S \gamma C_{\text {blood }} \mu_{\mathrm{a}}^{\mathrm{HbO}_{2}}(\lambda)+\left(1-\gamma C_{\text {blood }}\right) C_{\mathrm{H}_{2} \mathrm{O}} \mu_{\mathrm{a}}^{\mathrm{H}_{2} \mathrm{O}}(\lambda) \\
+\left(1-\gamma C_{\text {blood }}\right)\left(1-C_{\mathrm{H}_{2} \mathrm{O}}\right) \mu_{\mathrm{a}}^{(0)}(\lambda) .
\end{gathered}
$$

Here, $\mu_{\mathrm{a}}^{\mathrm{HbO}_{2}}(\lambda), \mu_{\mathrm{a}}^{\mathrm{Hb}}(\lambda)$ and $\mu_{\mathrm{a}}^{\mathrm{H}_{2} \mathrm{O}}(\lambda)$ are the absorption coefficients of oxy- and deoxyhaemoglobin and water, respectively, $C_{\text {blood }}$ and $C_{\mathrm{H}_{2} \mathrm{O}}$ are the layer volume fractions of blood and water contents (see table 2). We have calculated $\gamma$ assuming that haemoglobin is contained in the erythrocytes only, i.e.

$$
\gamma=F_{\mathrm{Hb}} F_{\mathrm{RBC}} H t
$$

where $H t$ is the haematocrit, $F_{\mathrm{RBC}}$ is the volume fraction of erythrocytes in the total volume of all blood cells and $F_{\mathrm{Hb}}$ is the volume fraction of haemoglobin in an erythrocyte. We presume that parameters $S, F_{\mathrm{RBC}}$ and $H t$ can change from layer to layer, in particular, due to the influence of various physiological factors (Renkin et al 1984, Priezzhev 2001). However, in the present simulation $F_{\mathrm{RBC}}=0.99, F_{\mathrm{Hb}}=0.25, S=0.6$ and $H t=0.45$ are the same for all the layers, and the values of other parameters, that we believe are typical for the normal skin and skin blood (Priezzhev 2001), are represented in table 2 .

The absorption coefficients: $\mu_{\mathrm{a}}^{\mathrm{Hb}}(\lambda), \mu_{\mathrm{a}}^{\mathrm{HbO}_{2}}(\lambda), \mu_{\mathrm{a}}^{\mathrm{H}_{2} \mathrm{O}}(\lambda)$ and $\mu_{\mathrm{a}}^{(0)}(\lambda)$ for the optical/NIR $(400-1100 \mathrm{~nm})$ are presented in figure 4. Here, oxy- and deoxyhaemoglobin and water absorption coefficients per mole were taken from a compilation of data (Jacques 1996, Prahl 1999), and the absorption spectrum of melanin $\mu_{\mathrm{a}}^{\operatorname{melanin}}(\lambda)$ is calculated as $5 \times 10^{9} \times \lambda^{-3.33}$.

Other skin layer optical properties used in the simulation, i.e. scattering coefficient $\mu_{\mathrm{s}}$, anisotropy factor $g$ and refractive index $n$, are also represented in table 2 . These data are collected from the literature: the scattering coefficients and anisotropy factors are taken from Jacques (1996), Simpson et al (1998), Doornbos et al (1999) and Tuchin (2000) and refractive indices from Tearney et al (1995), Gonzalez et al (1998) and Tuchin (2000). The values used correspond to a wavelength $\lambda=632.8 \mathrm{~nm}$. The refractive index of the ambient medium is taken to be $n_{0}=1$. 


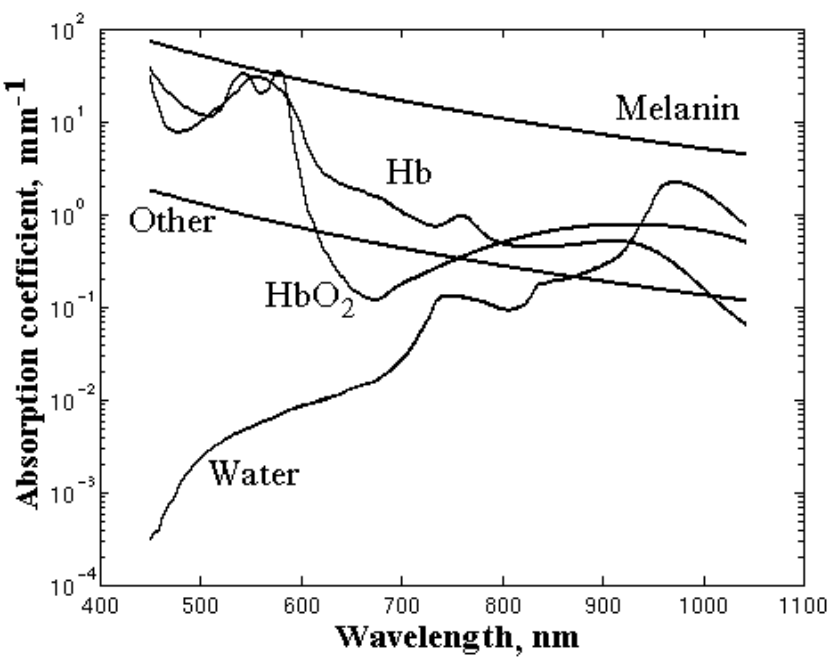

Figure 4. The absorption coefficients of oxy-, deoxyhaemoglobin and water as a function of wavelength. These data are taken from literature reports (Jacques 1996, Prahl 1999).

\section{Results and Discussion}

\subsection{Calculation of the skin layers absorption coefficients}

The absorption coefficients calculated by (10) for the skin layers containing blood (i.e. papillary dermis, upper blood net dermis, reticular dermis, deep blood net dermis and subcutaneous fat) are presented in figure 5. The absorption coefficients for blood-free layers, i.e. for stratum corneum and living epidermis, are calculated as

$\mu_{\mathrm{a}}^{\text {stratum }}(\lambda)=\left(\left(0.1-0.3 \times 10^{-4} \lambda\right)+0.125 \mu_{\mathrm{a}}^{(0)}(\lambda)\right)\left(1-C_{\mathrm{H}_{2} \mathrm{O}}\right)+C_{\mathrm{H}_{2} \mathrm{O}} \mu_{\mathrm{a}}^{\mathrm{H}_{2} \mathrm{O}}(\lambda)$

$\mu_{\mathrm{a}}^{\text {living epidermis }}(\lambda)=\left(C_{\text {melanin }} \mu_{\mathrm{a}}^{\text {melanin }}(\lambda)+\left(1-C_{\text {melanin }}\right) \mu_{\mathrm{a}}^{(0)}(\lambda)\right)\left(1-C_{\mathrm{H}_{2} \mathrm{O}}\right)+C_{\mathrm{H}_{2} \mathrm{O}} \mu_{\mathrm{a}}^{\mathrm{H}_{2} \mathrm{O}}(\lambda)$.

In the blood containing layers the absorption of oxy- and deoxyhaemoglobin dominates for the wavelength shorter than $600 \mathrm{~nm}$, whereas, the peaks at 970-980 nm featured in all layers, except stratum corneum, are produced by the water absorption (see figures 4 and 5 for comparison). It is well established that skin absorption in the visible and NIR spectral regions is determined by a combination of oxy-/deoxyhaemoglobin and water (Jacques 1996, Tuchin 2000). The results also agree well with experimental results for caucasian skin (Marchesini et al 1992), and show that absorption of the visible/NIR radiation in stratum corneum and living epidermis decreases uniformly with wavelength from 450 to $900 \mathrm{~nm}$.

\subsection{Simulation of skin reflectance spectra}

We have simulated skin reflectance spectra using the MC technique and with the optical properties and skin model described above. Typical results of the simulation (figure 6) show how the simulated skin reflectance spectra are sensitive to different fibre-optic spacing. In figure 6(a) we can see the response of the reflectance spectra for the different source-detector 


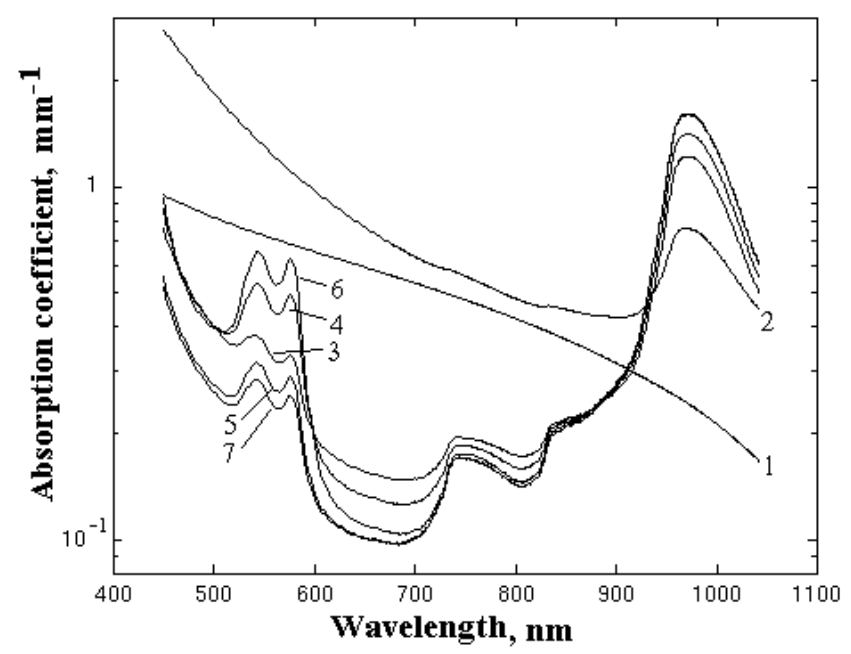

Figure 5. Simulated absorption coefficients of skin layers: 1-stratum corneum, 2-living epidermis, 3-papillary dermis, 4-upper blood net dermis, 5-reticular dermis, 6-deep blood net dermis and 7-subcutaneous fat.

separations when $C_{\text {blood }}$ of papillary and upper blood net dermis is changed from 0 to $40 \%$. The simulated reflectance spectra are not sensitive to changes of $C_{\text {blood }}$ in deep blood net dermis (figure 6(b)). This demonstrates that blood perfusion is important in the upper dermal layers. There are, however, also feeder vessels, which contribute to the optical appearance of the skin. The results of the simulation also illustrate what happens if the absorption properties of the upper skin layers are changed. These results agree well with the results of sampling volume studies (Meglinsky and Matcher 2001a, Meglinskii and Matcher 2001b), where it was shown that for small fibre-optic spacing $(200-800 \mu \mathrm{m})$, the main part of the detected signal becomes localized in the upper skin layers. It is important to note that the degree to which the signal is localized to a particular layer is a function of the absorption as well as scattering properties in the layer. An increase in the blood content in the upper layers will tend to reduce the overall path length in those layers; conversely a decrease in blood content will lengthen the path in them. However, we believe that the amount of blood used in our simulation (see table 2) is a reliable estimate for the true blood content in the skin tissues under normal conditions and that our results are therefore representative of the true spatial origin of the detected signal in normal skin.

We have found that the results of our simulation are remarkably similar to our experimentally measured skin spectra in the visible 450-600 nm (figure 7). The measured reflectance spectra of skin are obtained by the spectrophotometer system described in figure 1 . Here, we have presented the visible region only, as it produced the best agreement (see figure 7). The difference in the results of the simulation and the experimental data in the NIR spectral region could be explained by the choice of the optical properties of the skin layers, i.e. we simulated the skin reflection spectra by assuming that the scattering properties of all skin layers (i.e. $\mu_{\mathrm{s}}$ and $g$ ) are wavelength independent. In reality the scattering of skin tissues tends to monotonically decrease from 450 to $1100 \mathrm{~nm}$ (Marchesini et al 1992, Simpson et al 1998. Doornbos et al 1999), and the difference in scattering at 450-600 $\mathrm{nm}$ and $700-1100 \mathrm{~nm}$ ranges is significant. Also we did not include in the modelling the influence of various pigments such as melanin, bilirubin, NADH, $\beta$-carotene and other chromophores. Nevertheless, the results of the simulation show that when the model is supplied with reasonable optical properties, in 


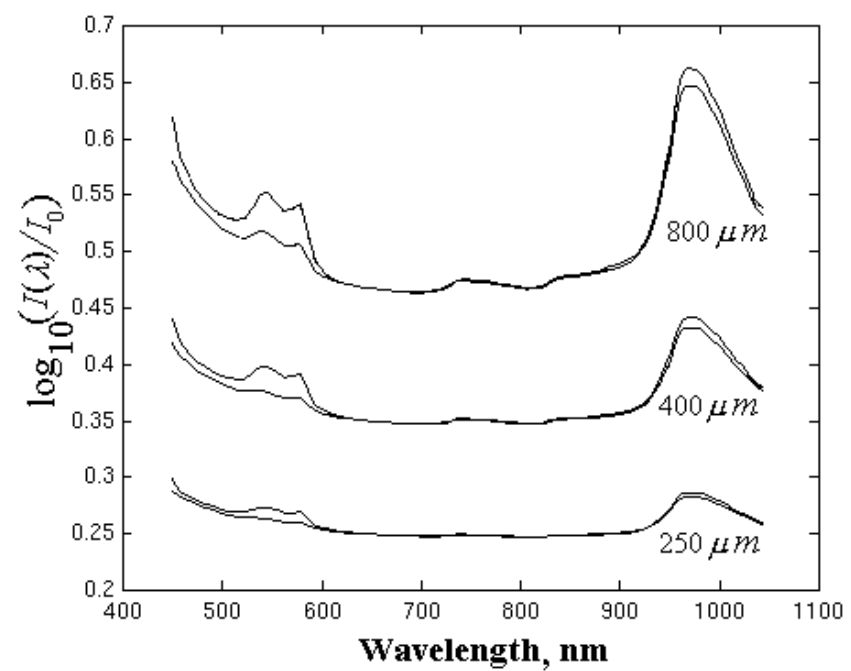

(a)

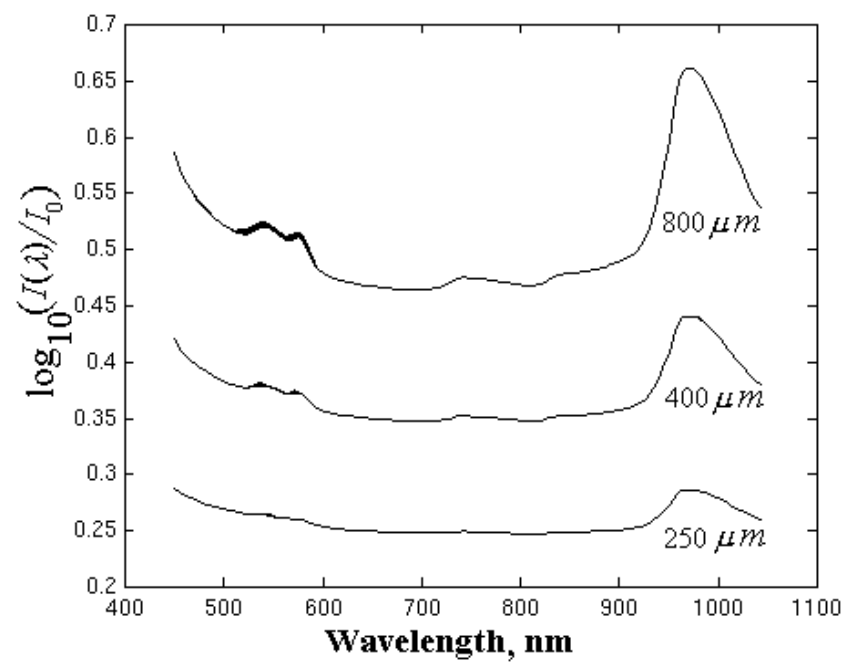

(b)

Figure 6. Skin reflectance spectra simulated: (a) for $40 \%$ blood volume fractions changes in papillary dermis and upper blood net dermis and (b) for $40 \%$ blood volume fractions changes in deep blood net dermis. Source and detector separations are: $250 \mu \mathrm{m}, 400 \mu \mathrm{m}$ and $800 \mu \mathrm{m}$. The assumed optical properties of the modelling medium are given in table 2 and in figure 5 .

the visible range, the results of the simulation agree reasonably well with the results of in vivo measurements.

\section{Summary}

We have developed a Monte Carlo model to predict the reflectance spectrum of skin, as measured by a fibre-optic probe of arbitrary geometry. The model allows for a spatially varying distribution of tissue absorber, such as haemoglobin, melanin and water, within anatomically distinct layers. By ignoring the slight wavelength dependence of tissue scattering, the effect of 


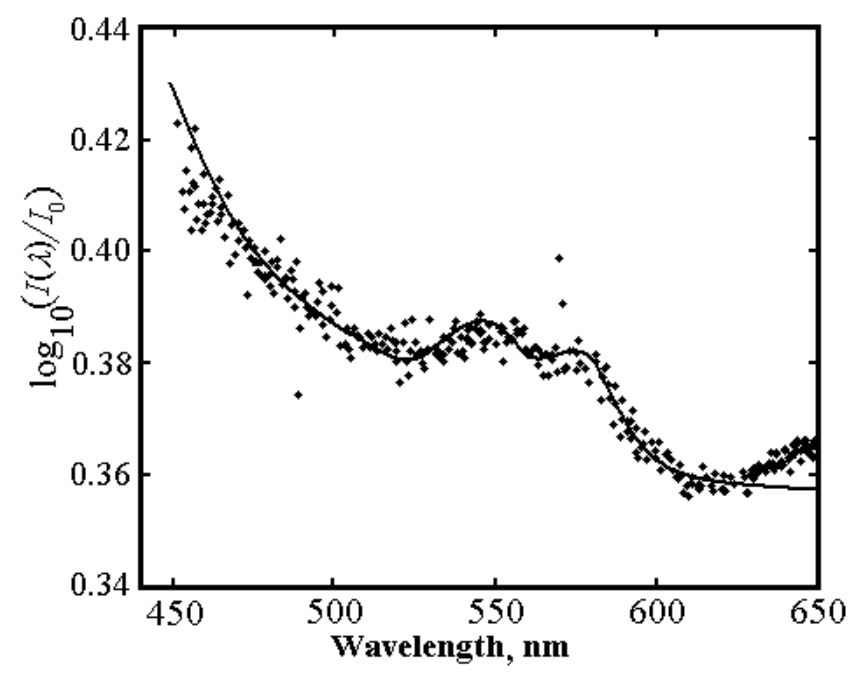

Figure 7. Skin reflectance spectra. Squares represent the results of MC spectra simulation and dots are the results of the skin reflectance spectrum measured in vivo.

a wavelength-dependent absorption coefficient can be calculated from a single MC calculation, allowing spectra to be calculated in a short time. We have simulated skin diffuse reflectance spectra by this MC technique assuming a wavelength-independent scattering coefficient for the different skin tissues and using the known wavelength dependence of the absorption coefficients of main skin tissue absorbers, such as oxy-/deoxyhaemoglobin, melanin and water. The absorption properties of skin tissues in the visible and NIR spectral region are estimated by taking into account the anatomical structure of skin as determined from histology, including the spatial distribution of blood vessels, water and melanin content. Reasonable estimates for oxygen saturation (based on likely metabolic demand) and haematocrit are also included, although these parameters are less well defined than the others as they cannot be determined from post-mortem samples. A number of clinical applications require the knowledge of tissues optical properties for in vivo express non-invasive tissue monitoring (Tuchin 2002, 2000). With this simulation we demonstrate the potential of the MC technique for use in studies of the oxygen saturation of blood, concentration of other skin chromophores, pigmentation, erythema and changes in skin colour. The algorithm used in the simulation allows one to save considerable time in the calculation of tissue reflectance spectra and yields results that agree reasonably well with in vivo measurements.

\section{Acknowledgments}

This work was supported in part by EPSRC grant GR/L89433. We thank Professor Angela Shore and Dr Paul Collier for useful and helpful discussions concerning human skin structure and its properties.

\section{References}

Bonner R F, Nossal R, Havlin S and Weiss G H 1987 Model for photon migration in turbid biological media J. Opt. Soc. Am. A 4 423-32

Chapman G 1980 The Body Fluids and their Functions (London: Edward Arnold) 
Corcuff P, Bertrand C and Leveque J L 1993 Morphometry of human epidermis in vivo by real-time confocal microscopy Arch. Dermatol. Res. 285 475-81

Cox A A and Durian D J 2001 Spatial sampling by diffuse photons Appl. Opt. 40 4229-35

Doornbos R M P, Lang R, Aalders M C, Cross F M and Sterenborg H J C M 1999 The determination of in vivo human tissue optical properties and absolute chromophore concentrations using spatially resolved steady-state diffuse reflectance spectroscopy Phys. Med. Biol. 44 967-81

Farrell T J, Patterson M S and Wilson B 1992 A diffusion theory model of spatially resolved, steady state diffuse reflectance for the noninvasive determination of tissue properties in vivo Med. Phys. 19 879-88

Flock S T, Patterson M S, Wilson B C and Wyman D R 1989 Monte Carlo modeling of light propagation in highly scattering tissue:I. Model predictions and comparison with diffusion theory IEEE Trans. Biomed. Eng. 36 $1162-8$

Gonzalez S, Rajadhyaksha M and Anderson R R 1998 Non-invasive (real-time) imaging of histologic margin of a proliferative skin lesion in vivo Int. Invest. Dermatol. $111538-9$

Henyey L G and Greenstein J L 1941 Diffuse radiation in the galaxy Astrophys. J. 93 70-83

Holbrook K A 1991 Structure and functions of the developing human skin Physiology, Biochemistry, and Molecular Biology of the Skin vol 1, ed L A Goldsmith (Oxford: Oxford University Press) pp 63-112

Ishimaru A 1978 Wave Propagation and Scattering in Random Media (New York: Academic)

Jacques S L 1996 Origins of tissue optical properties in the UVA, visible and NIR regions Advances in Optical Imaging and Photon Migration vol 2, ed R R Alfano and J G Fujimoto (Washington, DC: OSA) pp 364-70

Keijzer M, Jacques S L, Prahl S A and Welch A J 1989 Light distributions in artery tissue: Monte Carlo simulations for finite-diameter laser beams Laser Surg. Med. 9 148-54

Kienle A and Patterson M S 1997 Improved solutions of the steady-state and the time-resolved diffusion equations for reflectance from semi-infinite turbid medium J. Opt. Soc. Am. A 14 246-54

Lu J Q, Hu X H and Dong K 2000 Modeling of the rough-interface effect on a converging light beam propagating in a skin tissue phantom Appl. Opt. 39 5890-7

Marchesini R, Clemente C, Pignoli E and Brambilla M 1992 Optical properties of in vivo epidermis and their possible relationship with optical properties of in vivo skin J. Photochem. Photobiol. B: Biol. 16 127-40

Matcher S J 2002 Signal quantification and localization in tissue near-infrared spectroscopy Handbook of Optical Biomedical Diagnostics ed V V Tuchin (Bellingham, WA: SPIE Optical Engineering Press) ch 9

Meglinsky I V and Matcher S J 2001a Modelling the sampling volume for the skin blood oxygenation measurements Med. Biol. Eng. Comput. 39 44-50

Meglinskii I V and Matcher S J 2001b The analysis of spatial distribution of the detector depth sensitivity in multilayered inhomogeneous highly scattering and absorbing medium by the Monte Carlo technique Opt. Spectrosc. $91654-9$

Odland G F 1991 Structure of the skin Physiology, Biochemistry, and Molecular Biology of the Skin vol 1, ed L A Goldsmith (Oxford: Oxford University Press) pp 3-62

Okada E, Firbank M, Schweiger M, Arridge S R, Cope M and Delpy D T 1997 Theoretical and experimental investigation of near-infrared propagation in a model of the adult head Appl. Opt. 36 21-31

Potts R O 1985 Stratum corneum hydration: experimental techniques and interpretations of results J. Soc. Cosmet. Chem. 37 9-33

Prahl S A 1999 Optical Absorption of Hemoglobin Technical Report Oregon Medical Laser Center

Prahl S A, Keijzer M, Jacques S L and Welch A J 1989 A Monte Carlo model of light propagation in tissue SPIE Institute Series 5 102-11

Priezzhev A V 2001 Optics of blood and new optical sensing and diagnostic technique and instrumentation Munich European Biomedical Optics Short Course 101 (Bellingham, WA: SPIE Optical Engineering Press)

Renkin E M, Michel C C and Geiger S R 1984 Handbook of Physiology. Section 2: The Cardiovascular System. Microcirculation Part 1 vol IV (Bethesda, MD: American Physiological Society)

Ryan T J 1991 Cutaneous circulation Physiology, Biochemistry, and Molecular Biology of the Skin vol 2, ed L A Goldsmith (Oxford: Oxford University Press) pp 1019-84

Saidi I S 1992 Transcutaneous optical measurement of hyperbilirubinemia in neonates PhD Thesis Rice University, Houston, TX, USA

Serup J and Jemec G B E 1995 Non-Invasive Methods and the Skin (Boca Raton, FL: CRC Press) pp 83-131

Simpson C R, Kohl M, Essenpreis M and Cope M 1998 Near-infrared optical properties of ex vivo human skin and subcutaneous tissues measured using the Monte Carlo inversion technique Phys. Med. Biol. 43 2465-78

Sobol' I M 1974 The Monte Carlo Method (Chicago, IL: University of Chicago Press)

Stenn K S 1988 The skin Cell and Tissue Biology ed L Weiss (Baltimore, MD: Urban and Shwarzenberg) pp 541-72

Svaasand L O, Norvang L T, Fiskerstrand E J, Stopps E K S, Berns M W and Nelson J S 1995 Tissues parameters determining the visual appearance of normal skin and port-wine stains Laser Med. Sci. 10 55-65 
Tearney G J, Brezinski M E, Southern J F, Bouma B E, Hee M R and Fujimoto J G 1995 Determination of the refractive index of highly scattering human tissue by optical coherence tomography Opt. Lett. 20 $2258-60$

Tuchin V V 2000 Tissue Optics: Light Scattering Methods and Instruments for Medical Diagnosis (Bellingham, WA: SPIE Optical Engineering Press)

Tuchin V V 2002 Handbook of Optical Biomedical Diagnostics (Bellingham, WA: SPIE Optical Engineering Press) Verkruysse W, Lucassen G W and van Gemert M J C 1999 Simulation of color of port wine stain skin and its dependence on skin variables Laser Surg. Med. 25 131-9

Wang L, Jacques S L and Zheng L 1995 MCML—Monte Carlo modelling of light transport in multi-layered tissues Comput. Methods Programs Biomed. 47 131-46

Wilson B C and Adam G 1983 A Monte Carlo model for absorption and flux distributions of light in tissue Med. Phys. 10 824-830

Yaroslavsky I V and Tuchin V V 1992 Light transport in multilayed scattering media. Monte Carlo modelling Opt. Spectrosc. 72 934-9 